\title{
Existence and Uniqueness Theorems for the Neutron Transport Equation*
}

\author{
K. M. CASE \\ Department of Physics \\ The University of Michigan, Ann Arbor, Michigan \\ AND \\ P. F. ZWEIFEL \\ Department of Nuclear Engineering \\ The University of Michigan, Ann Arbor, Michigan
}

(Received 13 May 1963)

\begin{abstract}
In an attempt to understand the conditions under which the neutron transport equation has solutions, and the properties of those solutions, a number of existence and uniqueness theorems are proved. One finds that the properties of the solution are closely related to the boundedness of the source as well as to certain velocity-space integrals of the scattering kernel. Both time-dependent and time-independent equations are considered as are also the time-dependent and time-independent adjoint equations. Although only a very few of all possible existence and uniqueness theorems for these equations are considered here, the work may serve as a guide to the treatment of similar problems.
\end{abstract}

\section{INTRODUCTION}

$\mathrm{V}$ ARIOUS theorems concerning the existence and uniqueness of solution to the neutron transport equation have appeared in the literature. For example, Case ${ }^{1}$ has proved uniqueness for the one-speed, time-dependent equation under the assumption that the kernel is rotationally invariant. He has, in addition, shown that under the same assumptions the solution of the time-independent equation is unique if $c(\mathbf{r})$, the mean number of neutrons emitted per collision, is everywhere less than one. Olhoeft ${ }^{2}$ has considered the more general velocity-dependent case, and has shown, subject to the same restriction on $c$, that a unique, integrable solution exists for the time-independent equation. Davison $^{3}$ has made some rather general remarks concerning existence and uniqueness for the timedependent case, but has only outlined the methods of proof and has actually said little about the restrictions which must be imposed in order that the theorems be true.

Basically, the situation is the following: There are a number of possible restrictions which one can imagine might be applied to the cross sections and sources appearing in the neutron transport equation. For certain of these restrictions it is possible to prove that continuous solutions exist; for other sets of restrictions the solutions may not be continuous but still integrable functions of one

1 * Supforted in part by the Office of Naval Research, Defartment of the Navy, and the United States Atomic Energy Ccrrmission.

1 K. M. Case, Rev. Mod. Phys. 29, 651 (1957).

2 Jack E. Olhoeft, "The Doppler Effect for Non-Uniform Temperatures," University of Michigan Ph.D. Thesis (1962).

${ }^{3}$ B. Davison, Neutron Transport Theory (Oxford University Press, London, 1957). or more of the independent variables involved [i.e., position, velocity, and (in the time-dependent case) time]. For other restrictions, it may be impossible to prove anything. We have investigated a large number of possible restrictions which might reasonably be imposed upon the cross sections and sources, and investigated the existence of unique solutions for each case. In this way, we have tried to bring some order into the chaos of "obvious" or partially proved results which at the present exist in the literature.

In addition, we consider not only the transport equation but the time-dependent and time-independent "adjoint equations," and investigate the sufficient conditions that unique solutions exist for those equations. It turns out that there are many cases in which existence and uniqueness theorems can be proved for one or the other, but not both.

In Sec. II of this paper, we convert the transport and adjoint equations to integral equations in the usual manner. Then, in Sec. III, we discuss the restrictions on the sources and cross sections which will, for physical reasons, be applied in all cases.

Then, in Secs. IV and $\mathrm{V}$ we consider various existence and uniqueness theorems for the timedependent transport and adjoint equations. In Sec. VI we consider theorems for the time-independent equations (both transport and adjoint) and finally, in Sec. VII, we discuss certain "byproducts" of the theorems-a formula for the minimum critical size of a reactor and a proof that the time-dependence of the solutions of the time-dependent equations must obey certain restriotions.

We have made no attempt to be comprehensive 
in our treatment of the existence and uniqueness problem. Indeed, one can imagine virtually an infinite number of possible theorems similar to those which we prove. However, we have presented some of those which we feel have the most intrinsic interest and, in addition, demonstrate well the method of proof. In this way, if any of the conditions which we consider are not met in a particular problem, our work may serve as a guide to the correct treatment.

\section{INTEGRAL EQUATION FORMULATION}

\section{A. The Transport Equations}

The time-dependent transport equation is

$$
\begin{aligned}
& \partial \psi(\mathbf{r}, \mathbf{v}, t) / \partial t \\
& \quad+v(\boldsymbol{\Omega} \cdot \nabla+\sigma(\mathbf{r}, \mathbf{v})) \psi(\mathbf{r}, \mathbf{v}, t)=q(\mathbf{r}, \mathbf{v}, t) \\
& \quad+\int v^{\prime} \sigma\left(\mathbf{r}, \mathbf{v}^{\prime} \rightarrow \mathbf{v}\right) \psi\left(\mathbf{r}, \mathbf{v}^{\prime}, t\right) d^{3} v^{\prime},
\end{aligned}
$$

while, in the stationary limit, we have

$$
\begin{aligned}
& v(\boldsymbol{\Omega} \cdot \nabla+\sigma(\mathbf{r}, \mathbf{v})) \psi(\mathbf{r}, \mathbf{v}) \\
& \quad=q(\mathbf{r}, \mathbf{v})+\int v^{\prime} \sigma\left(\mathbf{r}, \mathbf{\nabla}^{\prime} \rightarrow \mathbf{v}\right) \psi\left(\mathbf{r}, \mathbf{v}^{\prime}\right) d^{3} v^{\prime}
\end{aligned}
$$

Here $\psi$ is the neutron angular density (i.e., the one-particle distribution function); $\mathbf{v}=v \boldsymbol{\Omega}$ is the neutron velocity; $\sigma(\mathbf{r}, \mathbf{v})$ is the total cross section, and the kernel $\sigma\left(\mathbf{r}, \mathbf{v}^{\prime} \rightarrow \mathbf{v}\right)$ is the cross section for a neutron of velocity $\nabla^{\prime}$ to be emitted into $d^{3} v$ about $\mathrm{v}$.

Equation (1) may be converted into intergal equations in the usual way, i.e., by introducing the Green's function of the left-hand side. This Green's function is well known." We obtain for the integral equation equivalent to (1a)

$$
\begin{aligned}
& \psi(\mathbf{r}, \mathbf{v}, t)=Q(\mathbf{r}, \mathbf{v}, t) \\
& +\int_{0}^{\prime} d t^{\prime} \int d^{3} v^{\prime} v^{\prime} \sigma\left(\mathbf{r}-\mathbf{v}\left(t-t^{\prime}\right), \mathbf{v}^{\prime} \rightarrow \mathbf{v}\right) \\
& \times \psi\left(\mathbf{r}-\mathbf{v}\left(t-t^{\prime \prime}\right), \mathbf{v}^{\prime}, t^{\prime}\right) \\
& \times \exp \left[-\int_{t^{\prime}}^{t} v \sigma\left(\mathbf{r}-\mathbf{v}\left(t-t^{\prime \prime}\right), \mathbf{v}\right) d t^{\prime \prime}\right],
\end{aligned}
$$

where

$$
\begin{aligned}
& Q(\mathbf{r}, \mathbf{v}, t)=\psi(\mathbf{r}-\mathbf{v} t, \mathbf{v}, 0) \\
& \times \exp \left[-\int_{0}^{t} v \sigma\left(\mathbf{r}-\mathbf{v}\left(t-t^{\prime}\right), \mathbf{v}\right) d t^{\prime}\right]
\end{aligned}
$$

K. M. Case, F. de Hoffmann, and G. Placzek, Introduction to the Theory of Neutron Diffusion (U. S. Government Printing Office, Washington, D. C., 1953).

$$
\begin{aligned}
& +\int_{0}^{t} d t^{\prime} q\left(\mathbf{r}-\mathbf{\nabla}\left(t-t^{\prime}\right), \mathbf{\nabla}, t^{\prime}\right) \\
& \times \exp \left[-\int_{t^{\prime}}^{t} v \sigma\left(\mathbf{r}-\mathbf{v}\left(t-t^{\prime \prime}\right), \mathbf{v}\right) d t^{\prime \prime}\right] .
\end{aligned}
$$

Actually, we shall prove theorems involving the esistence and uniqueness of solutions within a given volume of space $V$ bounded by a surface $S$ when the incoming (or, in the case of the adjoint equations, the outgoing) angular distribution is specified on $S$. The simplest way to modify Eq. (2) to describe this situation is to define $q(\mathrm{r}, \mathrm{v}, t)$, $\psi(\mathbf{r}, \mathbf{v}, 0), \sigma(\mathbf{r}, \mathbf{v})$, and $\sigma\left(\mathbf{r}, \mathbf{v}^{\prime} \rightarrow \mathbf{v}\right)$ to vanish for $\mathbf{r} \notin V$. Then the specified incident angular distribution $\psi_{1}\left(\mathrm{r}_{a}, \nabla, t\right)$ is replaced by a surface source $q_{s}\left(r_{s}, v, t\right)$ on $S$ in the usual manner $:^{4}$

$q_{s}\left(\mathrm{r}_{s}, \mathbf{v}, t\right)$

$$
\begin{aligned}
& =v\left|\mathbf{\Omega} \cdot \mathbf{n}_{0}\right| \psi_{i}\left(\mathbf{r}_{s}, \mathbf{v}, t\right), \quad \mathbf{\Omega} \cdot \mathbf{n}_{0}<0, \quad t>0, \\
& =0 \text { otherwise, }
\end{aligned}
$$

where $\mathbf{n}_{0}$ is the outward normal to $S$. Then Eq. (2) still applies [understanding the redefinitions of $q$, $\psi, \sigma$, and $\sigma\left(\mathbf{r}, \nabla^{\prime} \rightarrow \nabla\right)$ mentioned above] with an additional term $Q$, added to $Q$, Eq. (2b), to account for the surface source:

$$
\begin{aligned}
Q_{s}=\frac{1}{v} q_{s}\left(\mathbf{r}_{s}, \mathbf{v},\right. & \left.t-R_{s} / v\right) \\
& \times \exp \left[-\alpha\left(\mathbf{r}, \mathbf{r}-R_{s} \mathbf{\Omega}, \mathbf{v}\right)\right] .
\end{aligned}
$$

Here $\alpha$ is the usual optical path

$$
\alpha\left(\mathbf{r}, \mathbf{v}^{\prime}, \mathbf{v}\right)=\int_{0}^{R} d s \sigma\left(\mathbf{r}-s \frac{\mathbf{R}}{R}\right),
$$

where $R_{s}$ is the distance from $\mathbf{r}$ to the surface $S$ along the direction $-\Omega$ (See Fig. 1). Note: $R_{0}=$ $R_{s}(\mathrm{r}, \mathbf{\Omega})$.

Equation (2), thus modified, is the general integral equation formulation of the time-dependent neutron transport problem with which we shall be concerned. It is convenient to rewrite it in the somewhat more tractable form:

$$
\psi(\mathbf{r}, \mathbf{v}, t)=Q^{\prime}(\mathbf{r}, \mathbf{v}, t)+K \psi(\mathbf{r}, \mathbf{v}, t),
$$

where $K$ is the integral operator

FIa. 1.

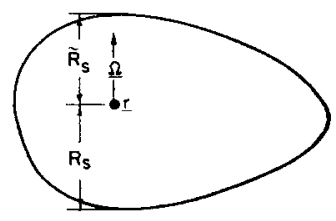


$K f(\mathbf{r}, \mathbf{\nabla}, t)$

$$
\begin{aligned}
= & \int_{0}^{t} d t^{\prime} \int d^{3} r^{\prime} \int d^{3} v^{\prime} \delta\left(\mathbf{r}^{\prime}-\mathbf{r}+\mathbf{v}\left(t-t^{\prime}\right)\right) \\
& \times \exp \left[-\int_{t^{\prime}}^{t} v \sigma\left(\mathbf{r}-\mathbf{v}\left(t-t^{\prime \prime}\right), \mathbf{v}\right) d t^{\prime \prime}\right] \\
& \times v^{\prime} \sigma\left(\mathbf{r}^{\prime}, \mathbf{v}^{\prime} \rightarrow v\right) f\left(\mathbf{r}^{\prime}, \nabla^{\prime}, t^{\prime}\right),
\end{aligned}
$$

and

$$
Q^{\prime}(\mathbf{r}, \mathbf{v}, t)=Q(\mathbf{r}, \mathbf{v}, t)+Q_{s}(\mathbf{r}, \mathbf{v}, t) .
$$

The integral equation formulation of the timeindependent transport equation (1b) is obtained in just the same way, i.e., by introducing the Green's function of the left side of Eq. (1b). We obtain

$$
\phi(\mathbf{r}, \mathbf{v})=Q^{\prime}(\mathbf{r}, \mathbf{v})+\Lambda \phi(\mathbf{r}, \mathbf{v}),
$$

where $\Lambda$ is the integral operator

$$
\begin{aligned}
& \Lambda f(\mathbf{r}, \mathbf{v})=\int_{0}^{R_{a}} d R \int d^{3} r^{\prime} \int d^{3} v^{\prime} \delta\left(\mathbf{r}^{\prime}-\mathbf{r}+R \mathbf{\Omega}\right) \\
& \times \exp \left[-\alpha\left(\mathbf{r}, \mathbf{r}^{\prime}, \mathbf{v}\right)\right] v^{\prime} \sigma\left(\mathbf{r}^{\prime}, \mathbf{v}^{\prime} \rightarrow \mathbf{v}\right) f\left(\mathbf{r}^{\prime}, \mathbf{v}^{\prime}\right), \quad(7 \mathbf{b}) \\
& Q^{\prime}(\mathbf{r}, \mathbf{v})=q_{s}\left(\mathbf{r}_{s}, \mathbf{v}\right) \exp \left[-\alpha\left(\mathbf{r}, \mathbf{r}-R_{s} \mathbf{\Omega}, \mathbf{v}\right)\right] \\
& \quad+\int_{0}^{R,} q(\mathbf{r}-R \boldsymbol{\Omega}, \mathbf{v}) \exp [-\alpha(\mathbf{r}, \mathbf{r}-R \mathbf{\Omega}, \mathbf{v})] d R,
\end{aligned}
$$

and

$$
\phi(\mathbf{r}, \mathbf{v})=v \psi(\mathbf{r}, \mathbf{v}) .
$$

Again we mention that the cross sections and sources have been defined to vanish for $r \notin V$ (or for $t<0$ ).

\section{B. The Adjoint Equations}

The time-dependent adjoint equation is defined to be

$$
\begin{aligned}
& \frac{\partial \tilde{\psi}(\mathbf{r}, \mathbf{v}, t)}{\partial t}+v(-\mathbf{\Omega} \cdot \nabla+\sigma(\mathbf{r}, \mathbf{\nabla})) \tilde{\psi}(\mathbf{r}, \mathbf{\nabla}, t) \\
& =\tilde{q}(\mathbf{r}, \mathbf{\nabla}, t)+v \int d^{3} v^{\prime} \sigma\left(\mathbf{r}, \mathbf{\nabla} \rightarrow \mathbf{\nabla}^{\prime}\right) \tilde{\psi}\left(\mathbf{r}, \nabla^{\prime}\right)
\end{aligned}
$$

while the time-independent adjoint equation is

$$
\begin{aligned}
v(-\mathbf{\Omega} \cdot \nabla+ & \sigma(\mathbf{r}, \mathbf{v})) \tilde{\psi}(\mathbf{r}, \mathbf{v})=\tilde{q}(\mathbf{r}, \mathbf{v}) \\
& +v \int d^{3} v^{\prime} \sigma\left(\mathbf{r}, \mathbf{v} \rightarrow \mathbf{v}^{\prime}\right) \Psi\left(\mathbf{r}, \mathbf{v}^{\prime}\right)
\end{aligned}
$$

The adjoint of a solution of Eqs. (1a) and (1b) will be taken to be the solution of Eqs. (8a) and (8b) subject to appropriate boundary conditions.
These boundary conditions are that the outgoing density on $S, \psi_{0}\left(\mathbf{r}_{s}, \nabla, t\right)$, rather than the incoming, will be specified. As before, we replace the boundary conditions by a surface source:

$$
\begin{aligned}
& \tilde{q}_{s}\left(\mathbf{r}_{s}, \mathbf{v}, t\right) \\
& =v\left|\boldsymbol{\Omega} \cdot \mathbf{n}_{0}\right| \psi_{0}\left(\mathbf{r}_{s}, \mathbf{v}, t\right), \quad \mathbf{\Omega} \cdot \mathbf{n}_{0}>0, \quad t>0, \\
& =0 \text { otherwise. }
\end{aligned}
$$

We now convert Eqs. (8) into integral equations just as for the case of transport equations, obtaining for the time-dependent equation

$$
\tilde{\psi}(\mathbf{r}, \mathbf{v}, t)=\widetilde{Q}^{\prime}(\mathbf{r}, \mathbf{v}, t)+\tilde{K} \psi(\mathbf{r}, \mathbf{v}, t),
$$

where $\tilde{K}$ is the integral operator

$$
\begin{aligned}
& \tilde{K} f(\mathbf{r}, \mathbf{v}, t) \\
& =v \int_{0}^{t} d t^{\prime} \int d^{3} v^{\prime} \int d^{3} v^{\prime} \delta\left(\mathbf{r}^{\prime}-\mathbf{r}-\mathbf{v}\left(t-t^{\prime}\right)\right) \\
& \quad \times \exp \left[-\int_{t^{\prime}}^{t} v \sigma\left(\mathbf{r}+\mathbf{v}\left(t-t^{\prime \prime}\right), \mathbf{v}\right) d t^{\prime \prime}\right] \\
& \quad \times \sigma\left(\mathbf{r}^{\prime}, \mathbf{v} \rightarrow \mathbf{v}^{\prime}\right) f\left(\mathbf{r}^{\prime}, \mathbf{v}^{\prime}, t^{\prime}\right)
\end{aligned}
$$

and

$\tilde{Q}^{\prime}(\mathbf{r}, \mathbf{v}, t)=\tilde{\psi}(\mathbf{r}+\mathbf{v} t, \mathbf{v}, \mathbf{0})$

$\times \exp \left[-\int_{0}^{t} v \sigma\left(\mathbf{r}+\mathbf{v}\left(t-t^{\prime \prime}\right), \mathbf{v}\right) d t^{\prime \prime}\right]$

$$
\begin{aligned}
& +\int_{0}^{t} d t^{\prime} \tilde{q}\left(\mathbf{r}+\mathbf{v}\left(t-t^{\prime}\right), \mathbf{v}, t^{\prime}\right) \\
& \times \exp \left[-\int_{t^{\prime}}^{t} v \sigma\left(\mathbf{r}+\mathbf{v}\left(t-t^{\prime \prime}\right), \mathbf{v}\right) d t^{\prime \prime}\right] \\
& +(1 / v) q_{s}\left(\mathbf{r}_{s}, \mathbf{\nabla}, t-\tilde{R}_{s} / v\right) \\
& \times \exp \left[-\alpha\left(\mathbf{r}, \mathbf{r}+\tilde{R}_{s} \mathbf{\Omega}, \mathbf{v}\right)\right] .
\end{aligned}
$$

As before, cross sections and the initial distribution are defined to vanish for $\mathrm{r} \notin V$. Also, $\tilde{R}_{\mathrm{s}}=\tilde{R}_{\mathrm{s}}(\mathbf{r}, \mathbf{\Omega})$ is the distance from $\mathbf{r}$ to the surface along the direction $+\boldsymbol{\Omega}$ (rather than along the direction $\boldsymbol{\Omega} \boldsymbol{\Omega}$ as in the case of the transport equation). See Fig. 1.

For the time-independent adjoint equation we obtain similarly

$$
\tilde{\psi}(\mathbf{r}, \mathbf{v})=(1 / v) \tilde{Q^{\prime}}(\mathbf{r}, \mathbf{v})+\tilde{\Lambda} \tilde{\psi}(\mathbf{r}, \mathbf{v}),
$$

where $\widetilde{\Lambda}$ is defined by

$$
\begin{gathered}
\tilde{\Lambda} f(\mathbf{r}, \mathbf{v})=\int_{0}^{\tilde{n}_{\bullet}} d R \int d^{3} r^{\prime} \int d^{3} v^{\prime} \delta\left(\mathbf{r}^{\prime}-\mathbf{r}-R \mathbf{\Omega}\right) \\
\quad \times \exp \left[-\alpha\left(\mathbf{r}, \mathbf{r}^{\prime}, \mathbf{v}\right)\right] \sigma\left(\mathbf{r}^{\prime}, \mathbf{v} \rightarrow \mathbf{v}^{\prime}\right) f\left(\mathbf{r}^{\prime}, \mathbf{v}^{\prime}\right),
\end{gathered}
$$

and 


$$
\begin{gathered}
\tilde{Q}^{\prime}(\mathbf{r}, \mathbf{v})=\tilde{q}_{s}\left(\mathbf{r}_{s}, \mathbf{v}\right) \exp \left[-\alpha\left(\mathbf{r}, \mathbf{r}+\tilde{R}_{s} \mathbf{\Omega}, \mathbf{v}\right)\right] \quad(11 \mathrm{c}) \\
\quad+\int_{0}^{\tilde{R}_{s}} \tilde{q}(\mathbf{r}+R \mathbf{\Omega}, \mathbf{v}) \exp [-\alpha(\mathbf{r}, \mathbf{r}+R \mathbf{\Omega}, \mathbf{v})] d R .
\end{gathered}
$$

\section{BASIC DEFINITIONS AND ASSUMPTIONS}

In all of our subsequent discussions we shall assume that the source functions $Q^{\prime}(\mathbf{r}, \mathbf{\nabla}, t)$ and $\widetilde{Q}^{\prime}(\mathbf{r}, \mathbf{\nabla}, t)$ as well as the cross sections $\sigma\left(\mathbf{r}, \mathbf{v}^{\prime} \rightarrow \mathbf{v}\right)$, and $\sigma(\mathbf{r}, \nabla)$, obey certain physically reasonable conditions.

(1) $Q^{\prime}$ and $\tilde{Q^{\prime}}$ are positive for all values of their arguments.

(2) Either $Q^{\prime}$ and $\widetilde{Q}^{\prime}$ are bounded or they can be written as the product of bounded functions multiplied by delta functions in one or more of their arguments.

(3) There exists a $v_{0}<\infty$ such that for $|\mathbf{v}|>v_{0}$, $Q^{\prime}$ and $\widetilde{Q}^{\prime}$ vanish identically.

(4) There exists a $v_{1}<\infty$ such that $\sigma\left(r, \nabla^{\prime} \rightarrow \nabla\right) \equiv 0$, for $v>v^{\prime}>v_{1}$.

Assumptions (3) and (4) permit us to avoid any difficulties that the infinite range of the velocity variable might otherwise introduce since together they imply that there are no neutrons present with speeds greater than $v_{\mathrm{m}}=\max \left(v_{0}, v_{1}\right)$. Then the integrals over $v^{\prime}$ in any of the integral equations can be written as

$$
\int d^{3} v^{\prime} \rightarrow \int d \boldsymbol{\Omega}^{\prime} \int_{0}^{v_{\mathrm{m}}}{v^{\prime 2}}^{2} d v^{\prime}
$$

These restrictions can actually be relaxed to some extent; we might assume that $Q^{\prime}$ and $\widetilde{Q^{\prime}}$ go to zero sufficiently rapidly as $v \rightarrow \infty$ that, if other restrictions are obeyed, the infinite range of $v$ need not cause any trouble. However, assumptions (3) and (4) are physically reasonable, and so we shall make no attempts to relax them.

(5) $\sigma\left(r, \nabla^{\prime} \rightarrow \nabla\right)$ can be written in the form

$$
\sigma\left(\mathbf{r}, \mathbf{v}^{\prime} \rightarrow \mathbf{v}\right)=\sum N_{i}(\mathbf{r}) \sigma_{i}\left(\mathbf{v}^{\prime} \rightarrow \mathbf{v}\right),
$$

where $N_{i}(\mathrm{r})$ is bounded. [Actually $N_{i}(\mathrm{r})$ represents a density of nuclei, and $\sigma_{i}$ is a microscopic crosssection, so we are merely assuming that there are no infinite concentrations of atoms present in the systems that we consider.]

(6) The cross-section $\sigma(\mathbf{r}, \quad \nabla)$ can be written in the same form:

$$
\sigma(\mathbf{r}, \mathbf{v})=\sum_{i} N_{i}(\mathbf{r}) \sigma_{i}(\mathbf{v}) .
$$

(7) $v \sigma_{i}(\nabla)$ is bounded. We expect $\sigma_{i}(v)$ to be bounded except possibly for $v \rightarrow 0$, in which case we admit $\sigma_{i}(v) \sim 1 / v$.
(8) $\sigma_{i}(\mathbf{r}, \mathbf{v}), \sigma\left(\mathbf{r}, \mathbf{v}^{\prime} \rightarrow \mathbf{v}\right), \sigma_{i}(\mathbf{v})$, and $\sigma_{i}\left(\mathbf{v}^{\prime} \rightarrow \mathbf{v}\right)$ are all positive.

(9) The function

$$
c(\mathbf{r}, \mathbf{v})=\int \sigma\left(\mathbf{r}, \mathbf{v} \rightarrow \mathbf{v}^{\prime}\right) d^{3} v^{\prime} / \sigma(\mathbf{r}, \mathbf{v})
$$

is positive. This assumption is also physically reasonable, since $c(r, v)$ represents the mean number of neutrons emitted per collision. We define a similar microscopic quantity $\xi_{i}(v)$ which is also bounded

$$
\xi_{i}(\mathbf{v})=\int \sigma_{i}\left(\mathbf{v} \rightarrow \mathbf{v}^{\prime}\right) d^{3} v^{\prime} / \sigma_{i}(\mathbf{v}) .
$$

We shall also have occasion to use two further functions,

$$
c^{\prime}(\mathbf{r}, \mathbf{v})=\int \sigma\left(\mathbf{r}, \mathbf{v}^{\prime} \rightarrow \mathbf{v}\right) d^{3} v^{\prime} / \sigma(\mathbf{r}, \mathbf{v}),
$$

and

$$
c^{\prime \prime}(\mathbf{r}, \mathbf{v})=\int v^{\prime} \sigma\left(\mathbf{r}, \mathbf{v}^{\prime} \rightarrow \mathbf{v}\right) d^{3} v^{\prime} / \sigma(\mathbf{r}, \mathbf{v})
$$

It may be noted that $c^{\prime}$ and $c^{\prime \prime}$ may not always be bounded but they (like $c$ ) are always positive. Similarly the functions

$$
\xi_{i}^{\prime}(\mathbf{v})=\int \sigma_{i}\left(\mathbf{v}^{\prime} \rightarrow \mathbf{v}\right) d^{3} v^{\prime} / \sigma_{i}(\mathbf{v})
$$

and

$$
\xi_{i}^{\prime \prime}(\mathbf{v})=\int v^{\prime} \sigma_{i}\left(\mathbf{v}^{\prime} \rightarrow \nabla\right) d^{3} v^{\prime} / \sigma_{i}(\mathbf{v})
$$

may not be bounded but, like $\xi_{i}(\mathbf{v})$, they are positive.

\section{THE TIME-DEPENDENT TRANSPORT EQUATION}

Bearing in mind the restrictions discussed in Sec. III, we consider now various existence and uniqueness theorems for the time-dependent transport equation.

Theorem 1. Let $Q^{\prime}(\mathbf{r}, \mathbf{v}, t)$ be bounded. Then if $c^{\prime \prime}(\mathbf{r}, \nabla)$ is bounded, a unique, positive, and continuous solution to the time-dependent transport equation exists.

We prove the theorem by constructing the Neumann series solution to Eq. (6):

$$
\psi(\mathbf{r}, \mathbf{\nabla}, t)=\sum_{n=0}^{\infty} \psi_{n}(\mathbf{r}, \mathbf{v}, t)
$$

where

$$
\psi_{0}(\mathbf{r}, \mathbf{\nabla}, t)=Q^{\prime}(\mathbf{r}, \mathbf{\nabla}, t)
$$


and

$$
\psi_{n}(\mathbf{r}, \mathbf{v}, t)=K \psi_{n-1}(\mathbf{r}, \mathbf{v}, t) .
$$

By hypothesis,

$$
0 \leqq \psi_{0} \leqq M<\infty .
$$

Furthermore,

$$
\begin{aligned}
\psi_{\mathbf{1}}(\mathbf{r}, \boldsymbol{\nabla}, t) & =K Q^{\prime}(\mathbf{r}, \boldsymbol{\nabla}, t) \\
& \leqq M c_{\max }^{\prime \prime} \sigma_{\max } t
\end{aligned}
$$

Continuing the iteration we find

$$
\psi_{n}(\mathbf{r}, \mathbf{v}, t) \leqq M\left(c_{\max }^{\prime \prime} \sigma_{\max }\right)^{n}\left(t^{n} / n !\right) .
$$

Thus, the Neumann series converges pointwise, and the theorem is proved. The fact that $\psi(r, \nabla, t)$ is positive follows from the fact that every term in the series is positive.

Next, assume that $c^{\prime \prime}(r, \nabla)$ is not bounded. Then we can state:

Theorem 2. Let $Q^{\prime}(\mathbf{r}, \mathbf{v}, t)$ be bounded. Then a unique, positive solution to the transport equation exists which is a continuous function of $t$ and $r$ and an integrable function of $\mathbf{v}$.

Proof: The proof proceeds along the lines of Theorem 1; however, it is sufficient to show that the series

$$
\sum_{n} \int d^{3} v \psi_{n}(\mathbf{r}, \mathbf{\nabla}, t)
$$

converges pointwise. Consider first

$$
\begin{aligned}
& \int d^{3} v \psi_{1}(\mathbf{r}, \mathbf{v}, t) \leqq M \int_{0}^{t} d t^{\prime} \int v^{\prime} d^{3} v^{\prime} \\
& \times \int d^{3} v \sigma\left(\mathbf{r}-\mathbf{\nabla}\left(t-t^{\prime}\right), \mathbf{v}^{\prime} \rightarrow \mathbf{\nabla}\right) .
\end{aligned}
$$

However, we have assumed in Sec. III that $\sigma(\mathbf{r}$, $\nabla^{\prime} \rightarrow \nabla$ ) could be written in the form

$$
\sigma\left(\mathbf{r}, \mathbf{v}^{\prime} \rightarrow \mathbf{v}\right)=\sum N_{i}(\mathbf{r}) \sigma_{i}\left(\mathbf{v}^{\prime} \rightarrow \mathbf{v}\right),
$$

where the $N_{i}(\mathbf{r})$ are bounded, say by $N_{i 0}$. Then

$$
\begin{aligned}
& \int d^{3} v \psi_{1}(\mathbf{r}, \mathbf{v}, t) \\
& \quad \leqq M t \sum N_{i 0} \int v^{\prime} d^{3} v^{\prime} \xi_{i}\left(\mathbf{v}^{\prime}\right) \sigma_{i}\left(\mathbf{v}^{\prime}\right) .
\end{aligned}
$$

But $\xi_{i}\left(v^{\prime}\right)$ and $\sigma_{i}\left(v^{\prime}\right)$ are assumed in Sec. III to be bounded, say by $\xi_{i 0}$ and $\sigma_{i 0}$. Then

$\int d^{3} v \psi_{1}(\mathbf{r}, \mathbf{\nabla}, t) \leqq M t \sum N_{i 0} \xi_{i 0} \sigma_{i 0} \int v^{\prime} d^{3} v^{\prime}$,

and since $v^{\prime} \leqq v_{m}$ (cf. Sec. III), we have

$$
\int d^{3} v \psi_{i}(\mathbf{r}, \mathbf{v}, t) \leqq K t, \quad 0 \leqq K<\infty .
$$

Similarly, we find

$\int d^{3} v \psi_{n}(\mathbf{r}, \nabla, t) \leqq \frac{K^{\prime} t^{n}}{n !}, \quad 0 \leqq K^{\prime}<\infty$,

and the theorem is proved. Again each term is positive, so that the solution is positive.

Next consider the case that $Q^{\prime}(\mathbf{r}, \nabla, t)$ is not bounded, but integrable. The following theorems are simple to prove by straightforward construction of the Neumann series, as above.

Theorem 3. If $Q^{\prime}(\mathbf{r}, \mathbf{v}, t)=Q_{0}(\mathbf{r}, \mathbf{v}) \delta(t)$, where $Q_{0}(r, v)$ is bounded, then, for the time-dependent transport equation:

(a) If $c^{\prime \prime}(\mathbf{r}, \mathbf{\nabla})$ is bounded, a unique, positive solution $\psi(\mathbf{r}, \nabla, t)$, exists. $\psi(\mathbf{r}, \nabla, t)$ is a continuous function of $\mathbf{r}$ and $\mathbf{v}$, and $\psi(\mathbf{r}, \nabla, t)-Q^{\prime}(\mathbf{r}, \mathbf{\nabla}, t)$ is a continuous function of $t$.

(b) If $c^{\prime \prime}(\mathbf{r}, \mathbf{v})$ is not bounded, then the solution $\psi(\mathbf{r}, \boldsymbol{\nabla}, t)$ may be an integrable rather than a continuous function of $v$. Otherwise the conclusions are unchanged.

Theorem 4. If $Q^{\prime}(\mathbf{r}, \mathbf{v}, t)=Q_{1}(\mathbf{r}, t) \delta\left(\mathbf{v}-\nabla_{0}\right)$, where $Q_{1}(\mathrm{r}, t)$ is bounded, then:

(a) If $v \sigma\left(\mathbf{r}, \mathbf{v}^{\prime} \rightarrow \mathbf{v}\right)$ is bounded, a unique, positive solution $\psi(\mathbf{r}, \nabla, t)$ exists. $\psi(\mathbf{r}, \nabla, t)$ is a continuous function $\mathbf{r}$ and $t$, and $\psi(\mathbf{r}, \mathbf{v}, t)-Q^{\prime}(\mathbf{r}, \mathbf{v}, t)$ is a continuous function of $\mathbf{v}$.

(b) If $v \sigma\left(\mathbf{r}, \mathbf{\nabla}^{\prime} \rightarrow \mathbf{\nabla}\right)$ is not bounded, then $\psi(\mathbf{r}, \mathbf{v}, t)-Q^{\prime}(\mathbf{r}, \mathbf{v}, t)$ may be an integrable rather than a continuous function of $\nabla$. [In proving part (b) we make use of the fact (cf. Sec. III) that $v$ and $\xi_{i}(\nabla)$ have both been assumed bounded.]

Theorem 5. If $Q^{\prime}(\mathbf{r}, \nabla, t)=Q_{2}^{\prime}(\mathbf{\nabla}, t) \delta\left(\mathbf{r}-\mathbf{r}_{0}\right)$, where $Q_{2}^{\prime}(r, t)$ is bounded, then:

(a) If $c^{\prime \prime}(\mathbf{r}, \mathbf{v})$ is bounded, a positive, unique solution $\psi(\mathbf{r}, \mathbf{v}, t)$ exists. $\psi(\mathbf{r}, \mathbf{v}, t)$ is a continuous function of $\mathbf{v}$ and $t$, and an integrable function of $\mathbf{r}$.

(b) If $c^{\prime \prime}(\mathbf{r}, \mathbf{v})$ is not bounded, then $\psi(\mathbf{r}, \mathbf{v}, t)$ may be an integrable rather than a continuous function of $\mathbf{v}$.

The theorems for the cases in which $Q^{\prime}(\mathbf{r}, \mathbf{\nabla}, t)$ involves a delta function in more than a single variable, may easily be constructed by appropriately combining the above theorems. We shall not state them separately.

\section{THE TIME-DEPENDENT "ADJOINT" EQUATION}

Here we deal with Eq. (10). The theorems will all be stated without proof, since the proofs are 
completely analogous to those given in the previous section.

Theorem 6. If $\tilde{Q}^{\prime}(\mathbf{r}, \mathbf{v}, t)$ is bounded, then a continouous, unique, positive solution exists. [Note that in proving this theorem it is necessary to make use of the fact that $c(\mathbf{r}, \mathbf{v})$ is bounded (cf. Sec. III).]

Theorem 7. If $\widetilde{Q}^{\prime}(\mathbf{r}, \mathbf{v}, t)=\widetilde{Q}_{0}(\mathbf{r}, \mathbf{v}) \delta(t)$, where $\widetilde{Q}_{0}$ is bounded, then a unique, positive solution exists. $\tilde{\psi}(\mathbf{r}, \mathbf{\nabla}, t)$ is a continuous function of $\mathbf{r}$ and $\mathbf{v}$, and $\tilde{\psi}(\mathbf{r}, \mathbf{v}, t)-\widetilde{Q}^{\prime}(\mathbf{r}, \mathbf{v}, t)$ is a continuous function of $t$.

Theorem 8. If $\widetilde{Q}^{\prime}(\mathbf{r}, \mathbf{v}, t)=\widetilde{Q}_{1}(\mathbf{r}, t) \boldsymbol{\delta}\left(\mathbf{v}-\mathbf{v}_{0}\right)$, where $\tilde{Q}_{1}$ is bounded, then:

(a) If $v \sigma\left(\mathbf{r}, \mathbf{v}^{\prime} \rightarrow \mathbf{v}\right)$ is bounded, a unique, positive solution $\tilde{\psi}(\mathbf{r}, \mathbf{v}, t)$ exists. $\tilde{\psi}(\mathbf{r}, \nabla, t)$ is a continuous function of $\mathbf{r}$ and $t$, and $\tilde{\psi}(\mathbf{r}, \mathbf{v}, t)-$ $\widetilde{Q}^{\prime}(\mathbf{r}, \mathbf{\nabla}, t)$ is a continuous function of $\mathbf{v}$.

(b) If $v \sigma\left(\mathbf{r}, \mathbf{v}^{\prime} \rightarrow \mathbf{v}\right)$ is unbounded but $\xi_{i}^{\prime \prime}(\mathbf{r}, \mathbf{v})$ is bounded, then the conditions above hold except that $\tilde{\psi}(\mathbf{r}, \mathbf{v}, t)-\widetilde{Q}^{\prime}(\mathbf{r}, \mathbf{v}, t)$ may be an integrable, rather than a continuous, function of $v$.

Theorem 9. If $\widetilde{Q}^{\prime}(\mathbf{r}, \mathbf{\nabla}, t)=\tilde{Q}_{2}(\mathbf{v}, t) \delta\left(\mathbf{r}-\mathbf{r}_{0}\right)$, where $\widetilde{Q}_{2}$ is bounded, then a unique, positive solution $\psi(\mathbf{r}, \mathbf{v}, t)$ exists. $\tilde{\psi}(\mathbf{r}, \mathbf{v}, t)$ is a continuous function of $\mathbf{v}$ and $t$, and an integrable function of $\mathbf{r}$.

Again, when $\widetilde{Q}^{\prime}$ involves delta functions in more than a single variable, the appropriate theorems can be constructed by appropriately combining the results above, and so we shall avoid stating them explicitly.

\section{THE TIME-INDEPENDENT TRANSPORT AND ADJOINT EQUATIONS}

In certain rather restrictive cases, it can be proved that unique, positive solutions of the timeindependent transport and adjoint equations exist. In general, the restrictions are much more severe than is the case for the time-dependent equations; we shall see later that this is to be expected.

Theorem 10. Let $Q^{\prime}(\mathbf{r}, \mathbf{v})$ be bounded and positive. Then if $c^{\prime}(\mathbf{r}, \mathbf{v})<1$, a unique, positive, and continuous solution to the time-independent transport equation [Eq. (7)] exists.

We note that the integral equation (7) differs from Eq. (1b) in that in the former, the dependent variable is the angular $f u x \phi(\mathbf{r}, \mathbf{v})$ rather than the angular density $\psi(\mathbf{r}, \mathbf{v})$. Thus all theorems which we shall prove involving the time-independent transport equation may not apply to the angular density unless $\psi(\mathbf{r}, \mathbf{v})$ vanishes sufficiently rapidly as $v \rightarrow 0$. This is not a real worry, since one is generally interested in the flux rather than the angular density.

As usual, we prove the theorem by constructing the Neumann series:

$$
\phi(\mathbf{r}, \mathbf{v})=\sum \phi_{n}(\mathbf{r}, \mathbf{v}),
$$

with

$$
\phi_{0}(\mathbf{r}, \mathbf{v})=Q^{\prime}(\mathbf{r}, v)
$$

and

$$
\phi_{n}(\mathrm{r}, \mathrm{v})=\Lambda^{n} \phi(\mathbf{r}, \mathrm{v})=\Lambda \phi_{n-1}(\mathrm{r}, \mathrm{v}) .
$$

By hypothesis, $\phi_{0}(r, \nabla)$ is bounded and positive:

$$
0 \leqq \phi_{0}(\mathrm{r}, \mathrm{v}) \leqq M<\infty .
$$

Next assume $\phi_{n-1}(\mathbf{r}, \mathbf{v})$ is bounded by $M^{\prime}$, say. Then

$$
\begin{aligned}
& \phi_{n}(\mathbf{r}, \mathbf{v})=\Lambda \phi_{n-1}(\mathbf{r}, \mathbf{v}) \leqq M^{\prime} c_{\max }^{\prime} \int_{0}^{\infty} d R \\
& \quad \times \exp [-\alpha(\mathbf{r}, \mathbf{r}-R \boldsymbol{\Omega}, \mathbf{v})] \sigma(\mathbf{r}-R \boldsymbol{\Omega}, \mathbf{v})
\end{aligned}
$$

The integral is easily shown to be equal to unity. Thus

$$
\phi_{n}(\mathbf{r}, \mathbf{v}) \leqq M^{\prime} c_{\max }^{\prime}<M^{\prime},
$$

since we have assumed $c^{\prime}(\mathbf{r}, \mathbf{v})<1$. This proves the theorem since the Neumann series converges pointwise, and each term is seen to be positive.

The next theorem is readily proved in essentially the same manner.

Theorem 11. If $Q^{\prime}\left(\mathbf{r}, \mathbf{v}=Q_{1}(\mathbf{r}, \mathbf{v}) \delta\left(\mathbf{v}-\mathbf{v}_{0}\right)\right.$, where $Q_{1}(\mathbf{r})$ is bounded, then if $c^{\prime}(\mathbf{r}, \nabla)<1$ and $\sigma\left(\mathbf{r}, \nabla_{0} \rightarrow \mathbf{v}\right)$ is bounded, a unique, positive solution $\psi(\mathbf{r}, \boldsymbol{\nabla})$ exists. $\psi(\mathbf{r}, \nabla)$ is a continuous function of $\mathbf{r}$ and $\psi(\mathbf{r}, \mathbf{v})-Q^{\prime}(\mathbf{r}, \mathbf{v})$ is a continouus function of $\mathbf{v}$.

Theorem 12. If $\sigma(\mathbf{r}, \mathbf{v}) Q^{\prime}(\mathbf{r}, \mathbf{v})$ is integrable, then if $c(\mathbf{r}, \mathbf{v})<1$, a unique, positive, integrable solution exists.

This is the theorem proved by Olhoeft. ${ }^{2}$ The procedure is to construct the Neumann series for the collision density $\chi(r, \nabla)$ defined by

$$
\chi(\mathbf{r}, \mathbf{v})=\sigma(\mathbf{r}, \mathbf{v}) \phi(\mathbf{r}, \mathbf{v}),
$$

and prove that

$$
\int d^{3} v d^{3} r K \chi_{n}(\mathbf{r}, \mathbf{v}) \leqq \int d^{3} r d^{3} v \chi_{n}(\mathbf{r}, \mathbf{v}) .
$$

Since the details are given in reference 2 , they will be omitted here. 
We next turn our attention to the time-independent adjoint equation. Here the theorems are quite analogous to Theorems 10-12 proved for the transport equation, except that the roles of $c$ and $c^{\prime}$ are reversed. Since the proofs are so similar, we simply state the theorems.

Theorem 13. Let $(1 / v) \tilde{Q}^{\prime}(\mathbf{r}, \mathbf{v})$ be bounded and positive. Then if $c(r, \nabla)<1$, a unique, positive, continuous solution exists to the time-independent adjoint equation.

Theorem 14. Let $(1 / v) \widetilde{Q}^{\prime}(\mathbf{r}, \mathbf{v})=\widetilde{Q}_{1}(\mathbf{r}) \delta\left(\mathbf{v}-\mathbf{v}_{0}\right)$, where $\tilde{Q}_{1}(\mathbf{r})$ is bounded and positive. Then if $c(r, \nabla)<1$ and $\sigma\left(r, \nabla \rightarrow \nabla_{0}\right)$ is bounded, a unique, positive solution $\tilde{\psi}(\mathbf{r}, \mathbf{v})$ exists. $\psi(\mathbf{r}, \mathbf{v})$ is a continuous function of $\mathrm{r}$ and $\tilde{\psi}-(1 / v) \tilde{Q}$ is a continuous function of $\mathbf{v}$.

Theorem 15. If $c^{\prime}(\mathbf{r}, \mathbf{v})<1$, and if $(1 / v) \sigma(\mathbf{r}, \mathbf{v}) \widetilde{Q}^{\prime}(\mathbf{r}, \mathbf{v})$ is integrable, then a unique, positive, integrable solution exists.

Note that in each of these theorems we have placed restrictions on $(1 / v) \tilde{Q}^{\prime}$ rather than on $\widetilde{Q}^{\prime}$. If we make the reasonable assumption that no zero-energy source neutrons are introduced into the system, then the conditions can equally well be stated as conditions on $\widetilde{Q}^{\prime}$ rather than on $(1 / v) \tilde{Q}^{\prime}$.

Next we prove a uniqueness theorem for cases in which existence has not been proved.

Theorem 16: If a solution of class $L^{2}$ of the timeindependent adjoint equation exists, then an $L^{2}$ solution to the time-independent transport equation, if it exists, will be unique.

We sketch the proof. Suppose there are two solutions to the transport equation, $\psi_{1}$ and $\psi_{2}$. Then $\psi \equiv \psi_{1}-\psi_{2}$ obeys the equation

$$
\begin{aligned}
& v\left(\mathbf{\Omega}^{\prime} \nabla+\sigma(\mathbf{r}, \mathbf{v})\right) \psi(\mathbf{r}, \mathbf{v}) \\
& \quad=\int v^{\prime} \sigma\left(\mathbf{r}, \mathbf{v}^{\prime} \rightarrow \mathbf{v}\right) \psi\left(\mathbf{r}, \mathbf{v}^{\prime}\right) d^{3} v^{\prime},
\end{aligned}
$$

with

$$
\psi\left(\mathbf{r}_{s}, \mathbf{v}\right)=0, \quad \mathbf{\Omega} \cdot \mathbf{n}_{0}<0 .
$$

Consider the adjoint equation with zero outgoing angular density:

$$
\begin{aligned}
& v(-\mathbf{\Omega} \cdot \boldsymbol{\nabla}+\sigma(\mathbf{r}, \mathbf{v})) \tilde{\psi}(\mathbf{r}, \mathbf{v}) \\
& =\tilde{q}(\mathbf{r}, \mathbf{v})+\int v \sigma\left(\mathbf{r}, \mathbf{v} \rightarrow \mathbf{v}^{\prime}\right) \tilde{\psi}\left(\mathbf{r}, \mathbf{v}^{\prime}\right) d^{3} v^{\prime}, \\
& \tilde{\psi}\left(\mathbf{r}_{s}, \mathbf{v}\right)=0, \quad \mathbf{\Omega} \cdot \mathbf{n}_{0}>0 .
\end{aligned}
$$

(We have hypothesized that such a solution exists.)
If we now multiply Eq. (37) by $\tilde{\psi}(\mathbf{r}, \mathbf{v})$, and multiply Eq. (39) by $\psi(\mathbf{r}, \nabla)$, subtract and integrate over $r$ and $v$, we obtain

$$
\begin{aligned}
& \int d^{3} v \int d S \boldsymbol{\Omega} \cdot \mathbf{n}_{0} \psi(\mathbf{r}, \mathbf{v}) \tilde{\psi}(\mathbf{r}, \mathbf{v}) \\
& =\int d^{3} r \int d^{3} v \int d^{3} v^{\prime} v^{\prime} \sigma\left(\mathbf{r}, \mathbf{v}^{\prime} \rightarrow \mathbf{v}\right) \\
& \times \psi\left(\mathbf{r}, \mathbf{v}^{\prime}\right) \tilde{\psi}(\mathbf{r}, \mathbf{v}) \\
& \quad-\int d^{3} r \int d^{3} v \int d^{3} v^{\prime} v \sigma\left(\mathbf{r}, \mathbf{v} \rightarrow \mathbf{v}^{\prime}\right) \Psi\left(\mathbf{r}, \mathbf{v}^{\prime}\right) \psi(\mathbf{r}, \mathbf{v}) \\
& \quad-\int d^{3} r \int d^{3} v \psi(\mathbf{r}, \mathbf{v}) \tilde{q}(\mathbf{r}, \mathbf{v}) .
\end{aligned}
$$

The left-hand side of this equation has been obtained with the help of Gauss' Theorem, and by virtue of Eqs. (38) and (40), it vanishes. Similarly, the first two terms on the right side of Eq. (41) cancel, and we obtain

$$
\int d^{3} r \int d^{3} v \psi(\mathbf{r}, \mathbf{v}) \tilde{q}(\mathbf{r}, \mathbf{v})=0
$$

Since $\tilde{q}(r, \nabla)$ is arbitary (and positive), it follows that

$$
\psi(\mathbf{r}, \mathbf{v}) \equiv 0,
$$

proving the theorem.

We note that the conditions of this theorem are satisfied for (among other cases) $c(r, \nabla)<1$; thus, Eq. (39) can possess no solution for $c(r, v)<1$ subject to the boundary conditions (38). Since these are precisely the equations satisfied by the neutron density in a reactor, we have succeeded in proving, as a byproduct, the not surprising result that a reactor cannot be critical if fewer neutrons are emitted than absorbed following each collision $(c<1)$. A simpler proof of this theorem (and of the following theorem) for the case that only scattering and pure absorption are present (i.e., no fission), is given in the Appendix.

The analog of Theorem (16) is:

Theorem 17. If an $L^{2}$ solution of the time-independent transport equation exists, then an $L^{2}$ solution of the time-independent adjoint equation, if it exists, will be unique.

The proof of this theorem is essentially identical with that of Theorem 16. The implication of this theorem is that a reactor cannot be critical if $c^{\prime}(r, v)<1$. This can be seen from a slight modification of the arguments used above to show the same result for $c(\mathbf{r}, \mathbf{v})<1$. 


\section{SOME MISCELLANEOUS RESULTS}

In the previous section we have pointed out that the uniqueness of the time-independent solution is equivalent to the statement that a reactor cannot be critical. Thus we have shown (Theorem 16) that no reactor can be critical for $c(\mathrm{r}, \mathrm{\nabla})<1$, which is, of course, physically obvious. However, such a condition is somewhat too stringent since it is clear that, for a system of finite size, neutron leakage may prevent a reactor from becoming critical even for $c$ somewhat larger than one.

We can obtain an estimate of the minimum value of $c$ for which a reactor of a given size will become critical from Theorem 12. The proof, which we omitted in Sec. VI, involves the construction of the Neumann series solution to the following integral equation for the collision density $\chi \equiv \sigma \phi$ :

$$
\chi(\mathbf{r}, \mathbf{v})=\sigma(\mathbf{r}, \mathbf{v}) Q^{\prime}(\mathbf{r}, \mathbf{v})+\tilde{\Lambda} \chi(\mathbf{r}, \mathbf{v}),
$$

where $\tilde{\Lambda}$ is the integral operator

$$
\begin{aligned}
& \tilde{\Lambda} f(\mathbf{r}, \mathbf{v})=\int_{0}^{R_{s}} d R \int d^{3} v^{\prime} v^{\prime} v d^{3} r^{\prime} \\
& \times \exp \left[-\alpha\left(\mathbf{r}, \mathbf{r}^{\prime}, \mathbf{v}\right)\right] \sigma(\mathbf{r}, \mathbf{v}) \delta\left(\mathbf{r}^{\prime}-\mathbf{r}+R \mathbf{\Omega}\right) \\
& \times \frac{\sigma\left(\mathbf{r}^{\prime}, \mathbf{v}^{\prime} \rightarrow \mathbf{v}\right)}{\sigma\left(\mathbf{r}^{\prime}, \mathbf{v}^{\prime}\right)} f\left(\mathbf{r}^{\prime}, \mathbf{v}^{\prime}\right) .
\end{aligned}
$$

In the proof, one shows that if

$$
\iint d^{3} v d^{3} r \chi_{n}(\mathbf{r}, \mathbf{v}) \leqq M
$$

then

$$
\iint d^{3} v d^{3} r \chi_{n+1}(\mathrm{r}, \mathrm{v}) \leqq c_{\max } M
$$

Actually, a somewhat stronger condition holds, since from Eq. (44b) it follows immediately that

$$
\begin{gathered}
\iint d^{3} v d^{3} r \Lambda \chi_{n+1}(\mathbf{r}, \mathbf{v})=\int d^{3} r \int d^{3} v \int d^{3} v^{\prime} \\
\times\left(1-\exp \left[-\alpha\left(\mathbf{r}, \mathbf{r}+R_{s} \mathbf{\Omega}, \mathbf{v}\right)\right]\right) \\
\times \sigma\left(\mathbf{r}, \mathbf{v}^{\prime} \rightarrow v\right) \frac{\chi_{n}\left(\mathbf{r}, \mathbf{v}^{\prime}\right)}{\sigma(\mathbf{r}, \mathbf{v})} .
\end{gathered}
$$

In obtaining (45b), the exponential in Eq. (46) was set equal to zero. If instead we approximate Eq. (46) by the expression

$$
\left(1-e^{-\bar{\tau}}\right) \bar{c} \iint \chi_{n}\left(\mathbf{r}, \mathbf{v}^{\prime}\right) d^{3} r d^{3} v^{\prime}
$$

we see that an approximate limit for the convergence of the Neuman series is

$$
\bar{c}<\left(1-e^{-\bar{\tau}}\right)^{-1} .
$$

Here $\bar{\tau}$ is the average optical chord length of the system and $\bar{c}$ is the average value of $c(\mathbf{r}, \mathbf{v})$.

This expression should give a rough estimate of critical size since one can write

$$
\bar{\tau}=\bar{\sigma} \bar{l},
$$

where $\bar{\sigma}$ is the average cross section and $\bar{l}$ is the average chord length $(4 \mathrm{~V} / \mathrm{S})$. The averages of $c$ and $\sigma$ are taken both with respect to $r$ and $\nabla$.

A second result which follows from the theorems proved in the earlier section is that the solution of the time-dependent transport or adjoint equations can increase no faster than exponentially if the source is of exponential order or less. Consider first the transport equation. Let us write the source in the form

$$
Q^{\prime \prime}(\mathbf{r}, \mathbf{\nabla}, t)=Q_{0}(\mathbf{r}, \nabla) f(t) .
$$

For simplicity we shall assume that $Q_{0}$ and $c^{\prime \prime}$ are bounded. Then we wish to examine the timedependence of the Neumann series, Eq. (19). Since $Q_{0}$ is bounded we have

$$
\begin{gathered}
\psi_{0} \leqq M f(t), \\
\psi_{1} \leqq M c_{\max }^{\prime \prime} \sigma_{\max } \int_{0}^{t} t f(t) d t,
\end{gathered}
$$

and in general

$$
\begin{aligned}
\psi_{n} \leqq & M\left(c_{\max }^{\prime \prime} \sigma_{\max }\right)^{n} \\
& \times \int_{0}^{t} d t_{1} \int^{t_{1}} d t_{2} \cdots \int_{0}^{t_{n-1}} t_{n} f\left(t_{n}\right) d t_{n} .
\end{aligned}
$$

Now from Euler's identity

$$
\begin{aligned}
\int_{0}^{t} d t_{1} \cdots \int_{0}^{t_{n-1}} t_{n} f\left(t_{n}\right) d t_{n} \\
=\int_{5}^{t} \frac{\left(t-t^{\prime}\right)^{n-1}}{(n-1) !} f\left(t^{\prime}\right) d t^{\prime},
\end{aligned}
$$

we have

$$
\psi_{n} \leqq M\left(c_{\max }^{\prime \prime} \sigma_{\max }\right)^{n} f_{\max }\left(t^{n} / n !\right)
$$

Thus

$$
\psi \leqq M f_{\max } \sum_{n=0}^{\infty} \frac{\left(c_{\max }^{\prime \prime} \sigma_{\max } t\right)^{n}}{n !}
$$

or

$$
\psi \leqq M f_{\max } \exp \left(c_{\max }^{\prime \prime} \sigma_{\max } t\right)
$$

Thus the time behavior of $\psi$ is asymptotically given either by $f(t)$ or by the exponential exp $\left(c^{\prime \prime}{ }_{\max } \sigma_{\max } t\right)$, whichever is more important at large times.

If $f(t)=e^{\alpha t}$, the integral in (54) can be evaluated explicitly: 


$$
\begin{aligned}
& I_{n}(t)=\int_{0}^{t} \frac{\left(t-t^{\prime}\right)^{n-1}}{(n-1) !} e^{\alpha t^{\prime}} d t^{\prime} \\
& \quad=e^{\alpha t} \int_{0}^{t} \frac{\tau^{n-1}}{(n-1) !} e^{-\alpha \tau} d \tau .
\end{aligned}
$$

Clearly,

$I_{n}(t)=\frac{e^{\alpha t}}{(n-1) !}(-1)^{n-1} \frac{\partial}{\partial \alpha^{n-1}}\left(\frac{1-e^{-\alpha t}}{\alpha}\right)$,

so that

$\psi_{n} \leqq M \frac{\left(c_{\max }^{\prime \prime} \sigma_{\max }\right)^{n}(-1)^{n-1}}{(n-1) !} \frac{\partial^{n-1}}{\partial \alpha^{n-1}}\left(\frac{1-e^{-\alpha t}}{\alpha}\right)$,

for $n>0$. Then

$$
\begin{aligned}
\psi & \leqq M\left[1+c_{\max }^{\prime \prime} \sigma_{\max } e^{\alpha t}\right. \\
& \left.\times \sum_{n=0} \frac{\left(c_{\max }^{\prime \prime} \sigma_{\max }\right)^{n}}{n !}(-1)^{n} \frac{\partial^{n}}{\partial \alpha^{n}}\left(\frac{1-e^{-\alpha t}}{\alpha}\right)\right],
\end{aligned}
$$

or

$$
\begin{aligned}
\psi \leqq M\left[1+c_{\max }^{\prime \prime} \sigma_{\max } /\left(\alpha-c_{\max }^{\prime \prime} \sigma_{\max }\right)\right. \\
\left.\times\left(e^{\alpha t}-e^{c^{\prime \prime \prime \max \sigma_{\max } t}}\right)\right],
\end{aligned}
$$

where Taylor's theorem has been used to perform the sum. We note that this limit is always positive.

A very similar argument can be used to delimit the time behavior of the adjoint equation; we shall omit it here.

\section{APPENDIX}

Theorems 16 and 17 may be proved in a different manner from that used in the text if we assume that no mechanism is present for neutron regeneration other than ordinary elastic and/or inelastic scattering. [We note that in this case $c(\mathbf{r}, \mathbf{v}) \leqq 1$.] In such a case, we expect that the kernel $\sigma\left(\mathbf{r}, \nabla^{\prime} \rightarrow \nabla\right)$ obeys the principle of detailed balance

$$
v^{\prime} M\left(v^{\prime}\right) \sigma\left(\mathbf{r}, \mathbf{v}^{\prime} \rightarrow \mathbf{v}\right)=v M(v) \sigma\left(\mathbf{r}, \mathbf{v} \rightarrow \mathbf{v}^{\prime}\right),
$$

since in the absence of sources and sinks of neutrons, an equilibrium should be approached. The equilibrium spectrum $M(v)$ is the Maxwell-Boltzmann distribution

$$
M(v) \sim v^{2} \exp \left[-\frac{1}{2}\left(M v^{2} / k T\right)\right] .
$$

Consider now Eq. (37) of the text, with the boundary condition, Eq. (38). Define a new dependent variable by the relation

$$
\phi(\mathbf{r}, \mathbf{v})=F(\mathbf{r}, \boldsymbol{\nabla}) \psi(\mathbf{r}, \boldsymbol{\nabla}),
$$

where $F(\mathbf{r}, \mathbf{v})$ is some well-behaved but otherwise arbitrary function to be chosen later. Then Eq. (37) becomes

$$
\nabla \cdot \nabla \frac{\phi}{F}+\frac{v \sigma \phi}{F}=\int v^{\prime} \sigma\left(\mathbf{v}^{\prime} \rightarrow \mathrm{v}\right) \frac{\phi\left(\mathbf{v}^{\prime}\right)}{F\left(\mathbf{v}^{\prime}\right)} d^{3} v^{\prime},
$$

with

$$
\phi\left(\mathbf{r}_{s}, \mathbf{v}\right)=\mathbf{0}, \quad \boldsymbol{\Omega} \cdot \mathbf{n}_{0}<0 .
$$

[The function $F$ must be chosen so that Eq. (A5) is still satisfied.]

If Eq. (A4) is multiplied by $\phi(r, v)$ and integrated over $r$ and $\nabla$ we obtain, with the aid of Gauss' Theorem,

$$
\begin{aligned}
& \frac{1}{2} \int d S \int d^{3} v \mathbf{n}_{0} \cdot \nabla \frac{\phi^{2}(\mathbf{r}, \mathbf{v})}{F(\mathbf{r}, \mathbf{v})} \\
& \quad+\int d^{3} r \int d^{3} v v \sigma(\mathbf{r}, \mathbf{v}) \frac{\phi^{2}(\mathbf{r}, \mathbf{v})}{F(\mathbf{r}, \mathbf{v})} \\
& \quad=\iint d^{3} v d^{3} v^{\prime} v^{\prime} \sigma\left(\mathbf{r}, \mathbf{v}^{\prime} \rightarrow \mathbf{v}\right) \frac{\phi(\mathbf{r}, \mathbf{v}) \phi\left(\mathbf{r}, \mathbf{v}^{\prime}\right)}{F\left(\mathbf{r}, \mathbf{v}^{\prime}\right)} .
\end{aligned}
$$

Next consider the identity

$$
\left[\phi(\mathbf{r}, \mathbf{v})-\phi\left(\mathbf{r}, \mathbf{v}^{\prime}\right)\right]^{2} \geqq 0,
$$

or

$$
\phi(\mathbf{r}, \mathbf{v}) \phi\left(\mathbf{r}, \nabla^{\prime}\right) \leqq \frac{1}{2}\left\{\phi^{2}(\mathbf{r}, \mathbf{v})+\phi\left(\mathbf{r}, \mathbf{v}^{\prime}\right)^{2}\right\} .
$$

Then, by virtue of (A8), the right side of Eq. (A6) may be written

$$
\begin{aligned}
& \iint d^{3} v d^{3} v^{\prime} v^{\prime} \sigma\left(\mathbf{r}, \mathbf{v}^{\prime} \rightarrow \mathbf{v}\right) \frac{\phi(\mathbf{r}, \mathbf{v}) \phi\left(\mathbf{r}, \mathbf{v}^{\prime}\right)}{F\left(\mathbf{r}, \mathbf{v}^{\prime}\right)} \\
& \leqq \\
& \frac{1}{2} \iint d^{3} v d^{3} v^{\prime} v^{\prime} \frac{\sigma\left(\mathbf{r}, \mathbf{v}^{\prime} \rightarrow \mathbf{v}\right)}{F\left(\mathbf{r}, \mathbf{v}^{\prime}\right)}\left\{\phi^{2}(\mathbf{r}, \mathbf{v})+\phi^{2}\left(\mathbf{r}, \mathbf{v}^{\prime}\right)\right\} \\
& =\frac{1}{2} \int d^{3} v^{\prime} v^{\prime} \sigma\left(\mathbf{r}, \mathbf{v}^{\prime}\right) c\left(\mathbf{r}, \mathbf{v}^{\prime}\right) \frac{\phi^{2}\left(\mathbf{r}, \mathbf{v}^{\prime}\right)}{F\left(\mathbf{r}, \mathbf{v}^{\prime}\right)} \\
& \quad+\frac{1}{2} \iint d^{3} v d^{3} v^{\prime} v^{\prime} \frac{\sigma\left(\mathbf{r}, \mathbf{v}^{\prime} \rightarrow \mathbf{v}\right)}{F\left(\mathbf{r}, \mathbf{v}^{\prime}\right)} \phi^{2}(\mathbf{r}, \mathbf{v}) .
\end{aligned}
$$

If we now choose $F\left(\mathbf{r}, \mathrm{v}^{\prime}\right)=1 / M\left(v^{\prime}\right)$, the second term on the right of Eq. (A10) becomes

$$
\begin{aligned}
& \frac{1}{2} \iint d^{3} v d^{3} v^{\prime} v^{\prime} M\left(v^{\prime}\right) \sigma\left(\mathbf{r}, \nabla^{\prime} \rightarrow \nabla\right) \phi^{2}(\mathbf{r}, \mathbf{v}) \\
& =\frac{1}{2} \iint d^{3} v d^{3} v^{\prime} v M(v) \sigma\left(\mathbf{r}, \mathbf{\nabla} \rightarrow \nabla^{\prime}\right) \phi^{2}(\mathbf{r}, \nabla),
\end{aligned}
$$

by Eq. (A1). This becomes, upon integration over $v^{\prime}$,

$$
\frac{1}{2} \int d^{3} v v M(v) \sigma(\mathbf{r}, \mathbf{v}) c(\mathbf{r}, \mathbf{v}) \phi^{2}(\mathbf{r}, \mathbf{v}) \text {. }
$$

writing $M(v)$ for $1 / F(\mathbf{r}, \nabla)$ throughout Eq. (A6), 
and using (A9), (A10), and (A12), we find

$$
\begin{aligned}
& \frac{1}{2} \int d S \int d^{3} v \mathrm{n}_{0} \cdot \mathrm{v} \phi^{2}(\mathrm{r}, \mathrm{v}) M(v) \\
& \quad+\int d^{3} r \int d^{3} v v M(v) \sigma(\mathrm{r}, \mathrm{v}) \phi^{2}(\mathrm{r}, \mathrm{v}) \\
& \quad-\int d^{3} v v \sigma(\mathrm{r}, \mathrm{v}) c(\mathrm{r}, \mathrm{v}) v M(v) \phi^{2}(\mathrm{r}, \mathrm{v}) \leqq 0 .
\end{aligned}
$$

The first term on the left side of Eq. (A13) is, by virtue of (A5), nonnegative. The other two terms combine to give

$\int d^{3} r \int d^{3} w M(v) \sigma(\mathbf{r}, \nabla) \phi^{2}(\mathbf{r}, \mathbf{v})[1-c(\mathbf{r}, \mathbf{v})]$,

which is always nonnegative since $c(\mathbf{r}, \mathbf{v})<1$. However, Eq. (A13) tells us that the sum of these terms is nonpositive. Thus

$$
\phi(\mathbf{r}, \mathbf{\nabla}) \equiv 0,
$$

and the theorem is proved. We see that a reactor with no fuel can never be critical.

The uniqueness of the time-independent adjoint equation for the case of no reproduction can be proved in an entirely analogous fashion. This method is a slight generalization of that used in reference 1 for proving uniqueness of the one-speed equations.

\section{ACKNOWLEDGMENTS}

One of the authors (P. F. Z.) is grateful for stimulating discussions with Professors Charles Dolph, R. K. Osborn, and Professor F. C. Shure.

\title{
Fundamental Properties of Perturbation-Theoretical Integral Representations. II
}

\author{
Noboru Nakanishi* \\ Institute for Advanced Study, Princeton, New Jersey \\ (Received 19 June 1963)
}

\begin{abstract}
In the first part of this paper, it is investigated, apart from theperturbation-theoreticalbasis, under what conditions the perturbation-theoretical integral representations can be derived, and two theorems are given concerning this problem. In the second part, the asymptotic behavior of the weight function in the integral representation is investigated in perturbation theory. It is proved that the weight function vanishes at infinity for an infinite sum over certain graphs which are much more general than the ladderlike graphs. This result gives the analyticity in the right half-plane of complex angular momentum.
\end{abstract}

\section{INTRODUCTION}

$I^{\mathrm{I} a}$ $\mathrm{N}$ a previous paper, ${ }^{1}$ which is quoted as $\mathrm{I}$, we have investigated various analyticity and uniqueness properties of the perturbation-theoretical integral representations. The purpose of the present paper is to make some further investigation on analytic properties and to examine the asymptotic behavior of weight functions.

In Sec. II the Deser-Gilbert-Sudarshan-Ida (DGSI)-type integral representation ${ }^{2}$ is investigated.

* Present address: Brookhaven National Laboratory, Upton, Long Island, New York.

1 N. Nakanishi, Phys. Rev. 127, 1380 (1962).

2 S. Deser, W. Gilbert, and E. C. G. Sudarshan, Phys. Rev. 115, 731 (1959). M. Ida, Progr. Theoret. Phys. (Kyoto) 23, 1151 (1960). See also N. Nakanishi, Progr. Theoret. Phys. (Kyoto) Suppl. 18, 1 (1961), Part III.
The conditions under which it can be derived are given in a practically more convenient form than that given in I.

The integral representation for the scattering amplitude ${ }^{3}$ consists of three terms, each of which is nothing but the DGSI-type integral representation for two variables among $s, t$, and $u$. Hence the analyticity domain $D$ of the former is the intersection of those of the latter. In Sec. III it is proved that a function holomorphic in $D$ can always be written as a sum of three functions holomorphic in the analyticity domain of the DGSI-type integral

3 N. Nakanishi, Progr. Theoret. Phys. (Kyoto) 26, 337, ${ }_{927}^{3}$ N. Na 1961$)$ 